\title{
CIENCIA EN CHILE EN CINCO MOMENTOS
}

\author{
Rafael Benguria Donoso
}




\section{RAFAEL BENGURIA DONOSO}

Premio Nacional de Ciencias Exactas 2005. Es Ingeniero Civil Electricista y Magíster en Física de la Universidad de Chile. Doctor en Física por la Universidad de Princeton y miembro de número de la Academia Chilena de Ciencias. Ha sido dos veces presidente de la Sociedad Chilena de Física. Actualmente se desempeña como académico en la Facultad de Física de la Pontificia Universidad Católica de Chile. 


\section{CIENCIA EN CHILE EN CINCO MOMENTOS}

Es un honor y un agrado participar en la celebración del cincuentenario de la Facultad de Ciencias de la Universidad de Chile y, en particular, hacerlo con un ensayo en los Anales de la Universidad de Chile, una revista académica que ha jugado un papel muy importante en la historia de nuestro país. Ingresé a la Universidad de Chile en marzo de 1968 a estudiar Ingeniería, solo tres años después de la creación de la Facultad de Ciencias, y he conocido a varios de sus profesores y alumnos a lo largo de su historia, muchos de los cuales han hecho contribuciones importantes a nuestro desarrollo científico. Por eso es particularmente grato unirme a esta celebración.

En este ensayo he querido describir mi visión, particular por cierto, del desarrollo científico de Chile (ver también [1]) y de los desafíos que tenemos por delante. He organizado esta presentación en cinco partes, definidas un poco arbitrariamente, que reflejan distintas situaciones del desarrollo científico chileno.

\section{EL ENTUSIASMO INICIAL}

Durante la Colonia el desarrollo de las ciencias físicas y matemáticas fue muy pobre o casi inexistente. En el siglo XVIII había en Santiago varios Reales Colegios, siendo los más importantes el Real Colegio Seminario del Santo Ángel de la Guarda y el Real Convictorio de San Francisco Javier, erigido por los jesuitas en 1611. Estuvo dirigido por los jesuitas hasta que la orden fue expulsada en 1767. Desde 1768 y hasta 1815 pasó a llamarse Real Colegio de San Carlos o Convictorio Carolino de Nobles. La fundación de una universidad en Chile tardó demasiado tiempo debido más bien a conflictos territoriales entre domínicos y jesuitas. Finalmente se fundó la Universidad Real de San Felipe en 1747, la cual en el papel contenía programas de matemáticas, pero que casi nunca se llevaron a cabo. El desarrollo de las matemáticas hacia el final del siglo XVIII era muy pobre en Chile. Esto empezó a cambiar en los años previos y posteriores a la Primera Junta de Gobierno, junto a las ideas de la Ilustración Europea. Manuel de Salas, en 1797, fundó la Academia de San Luis, que tuvo los primeros visos de instrucción en matemáticas.

En nuestros primeros doscientos años como Estado independiente ha habido distintos esfuerzos con miras a desarrollar nuestra capacidad científica. Los primeros 20 años, luego de la Primera Junta de Gobierno de 1810, un período extremadamente complejo, marcado por profundas divisiones que culminaron con la sangrienta 
Batalla de Lircay, no correspondieron a un tiempo propicio para desarrollar políticas de desarrollo cultural o científico. Por cierto fue una etapa de mucho entusiasmo, en la que abundaron sueños y esperanzas. Debido principalmente a la formación académica de Martínez de Rozas, quien antes de participar en la Independencia de Chile obtuvo su doctorado en la Universidad de Córdoba y ejerció como profesor de Física en Concepción, una de las primeras medidas de la Junta fue la creación de gabinetes de física en todos los establecimientos de enseñanza a lo largo del país.

Otra muestra de este entusiasmo inicial fue el editorial del número 9, del jueves 9 de abril de 1812, de la "Aurora de Chile", titulado "Educación". Se trataba de un extenso ensayo sobre el tema, cuya redacción ha sido usualmente atribuida a Juan Egaña. En parte de dicho ensayo se dice:

... "La práctica de las ciencias sólidas y el cultivo útil de los talentos es inseparable de la grandeza y felicidad de los estados. No es el número de los hombres el que constituye el poder de la nación, sino sus fuerzas bien arregladas, y estas provienen de la solidez, y profundidad de sus entendimientos. Cuando ellos saben calcular las relaciones que tienen las cosas entre sí, conocer la naturaleza de los entes, adquirir nuevas fuerzas con la mecánica, gobernar las familias y los pueblos con la política y la economía, saben también dirigir todas sus miras a un punto común y servirse de todos modos de la naturaleza..."

... "El hombre es un ente real y necesita de sólidos y prácticos conocimientos para vivir bien, no de ideas fantásticas, ni palabras buecas y sin sentido; y por esto se ve que las naciones que se versan en la buena física, en la historia natural, en la geometría, en la mecánica y en otras muchas pertenecientes al hombre físico, y que estudian la ética, la política y otras ciencias, por lo que respecta al hombre moral, nos llevan grandes ventajas en la ilustración y la sabiduría..."

Las acciones más importantes y duraderas luego de la Primera Junta de Gobierno, en este período inicial, fueron la fundación del Instituto Nacional y de la Biblioteca Nacional, a escasos días una de otra, en agosto de 1813. Estas dos instituciones han sido pilares de nuestro desarrollo cultural.

\section{HACIA UN DESARROLLO INSTITUCIONAL}

Durante mis años de estudiante de ingeniería en la Facultad de Ciencias Físicas y Matemáticas de la Universidad de Chile viví cerca de la Plaza Brasil y todos los días caminaba a la Escuela de Ingeniería atravesando distintas calles que de cierto modo reflejan el segundo período de este ensayo: calles como Lorenzo Sazié, Manuel Julián Grajales, Andrés Antonio Gorbea, Claudio Gay e Ignacio Domeyko recuerdan el 
período en que Chile empezó a sentar sus bases institucionales como república. Fue Mariano Egaña uno de los actores de este período de institucionalización. Hijo de Juan Egaña, autor del ensayo antes mencionado y principal redactor de la Constitución Política de Chile de 1833, Mariano Egaña fue quien dio los primeros pasos de la institucionalidad de la educación superior en Chile.

Comisionado como embajador plenipotenciario de Chile en Europa, Mariano Egaña convenció al ingeniero español radicado en París, Antonio Gorbea, de venir a Chile como profesor del Instituto Nacional (1826). Posteriormente Gorbea fue el primer decano de la Facultad de Ciencias Físicas y Matemáticas de la Universidad de Chile (1842). Fue Gorbea el que planificó la enseñanza de las matemáticas y de la física de los primeros ingenieros de la Universidad de Chile. En 1845 la Imprenta de la Opinión de Santiago publicó el Curso Completo de Matemáticas Puras de L. R. Francoeur, traducido y ampliado por Antonio Gorbea, que contenía gran parte de los contenidos de matemáticas que por mucho tiempo se enseñaron en la Escuela de Ingeniería de la Universidad de Chile. En su labor como embajador plenipotenciario Mariano Egaña también convenció a Andrés Bello, a la sazón en Londres, de venir a Chile (1829). Hacia fines de la segunda década del siglo XIX se fundaron en Santiago dos instituciones de enseñanza que tuvieron una muy corta vida: el Liceo de Chile y el Colegio de Santiago. En 1828 el comisionado por el gobierno chileno, el periodista francés Pedro Chapuis, convenció al naturalista Claudio Gay de venir a Chile (1828) y de servir como profesor del Colegio de Santiago, al cual se integró también Andrés Bello. Andrés Bello fue luego el fundador y primer rector de la Universidad de Chile (1842).

En el ámbito científico, durante el siglo XIX se realizaron grandes expediciones científicas por todo el globo. En este espíritu se enmarca la expedición de Fitz Roy y Darwin en el Beagle, y también la gran labor como naturalista de Claudio Gay, quien escribió su obra fundacional y monumental, "Historia Física y Política de Chile", y su "Atlas de la Historia Física y Política de Chile", recientemente reeditados (en 30 volúmenes) como parte de la Biblioteca Fundamentos de la Construcción de Chile por la DIBAM, la Pontificia Universidad Católica de Chile y la Cámara Chilena de la Construcción como una contribución al bicentenario de nuestra independencia.

Por su parte, Ignacio Domeyko, geólogo e ingeniero de minas nacido en Polonia y educado en París, fue contratado en 1838 por el Liceo de La Serena, por intermedio del industrial minero Carlos Lambert, para dar clases de química y de mineralogía. Aprovechó su tiempo en el Norte Chico para hacer extensas exploraciones geológicas. En 1847 se trasladó a Santiago y se integró como profesor en el Instituto Nacional. Fue luego contratado como parte del claustro académico de la Universidad de Chile en 1867 (en la Facultad de Filosofía y Educación) y el mismo año fue elegido rector de la Universidad, cargo en el que sirvió hasta 1883. 
La Universidad de Chile fue fundada en 1842 como una Academia de Ciencias, a la usanza de las Academias de Ciencia europeas, con un claustro académico determinado y sin labores docentes. La enseñanza superior se seguía impartiendo en el Instituto Nacional. Fue Ignacio Domeyko quien transformó la Universidad de Chile, uniendo en ella las actividades propias de investigación y docencia superior. (Un análisis más detallado del desarrollo institucional de las Universidades en Chile se puede ver en [6]).

\section{¿CIENCIA, ECONOMÍA Y DESARROLLO?}

Durante la década de los setenta del siglo XIX Chile vivió una profunda crisis económica. Luego de haber tenido un boom económico en las décadas anteriores, producido por las exportaciones de granos a California y Australia durante la fiebre del oro, las exportaciones chilenas cayeron bruscamente. Esto dio paso a una delicada situación económica en los 70 . Esto produjo gran inquietud entre los ingenieros de la época, los que en distintas tribunas abogaron por desarrollar la actividad industrial en Chile para obviar este tipo de crisis económicas. En medio y con posterioridad a la crisis se crearon instituciones como la Sociedad de Fomento Fabril (1883), el Instituto de Ingenieros (1888) y las Sociedades Científicas de Chile (1891) (ver e.g., [2]). Uno de los principales impulsores de estas tres instituciones fue Luis Zegers, académico de la Facultad de Ciencias Físicas y Matemáticas de la Universidad de Chile. Luis Zegers y otros integrantes de estas tres instituciones (SOFOFA, Instituto de Ingenieros y la Sociedad Científica de Chile) escribieron numerosos e ilustrados ensayos sobre la importancia de la ciencia y de la tecnología en el desarrollo económico y social de los países. Luis Zegers escribió numerosos ensayos científicos tanto en los Anales de la Universidad de Chile como en las Actas de la Sociedad Científica de Chile. Esta última fue el órgano oficial de la Sociedad Científica de Chile y fue publicada por más de 40 años desde 1891 [3].

Aunque no hay muchos artículos de física, de matemáticas o de astronomía en las Actas de la Sociedad Científica de Chile, sí hay algunos artículos bien notables para su época. Ciertamente el artículo más notable aparecido en las Actas es el artículo de Luis Zegers y Arturo Salazar (pp. 21-22, Tomo 6, 1896(1897)), en el que reportaron sobre la primera radiografía tomada por ellos en Chile, en marzo de 1896, pocos meses después del descubrimiento de los rayos $\mathrm{X}$ por Roentgen hacia fines de 1895. El astrónomo Albert Obrecht es autor de varios artículos aparecidos en las Actas, algunos sobre determinación de órbitas de planetas y cometas y otros bien interesantes que contienen, en entregas sucesivas, un curso completo de mecánica racional. Uno de los artículos más destacables de este período fue el de Luis Zegers, "La Constitución de la Materia", Anales de la Universidad de Chile, vol. 130, pp. 
151-176 (1912), que contiene una versión extendida de una Conferencia dada por Zegers en el Salón de Honor de la Universidad de Chile en noviembre de 1911. En este artículo Zegers describía varios resultados recientes de la física de esos años. En particular describía los experimentos de Joseph John Thomson que llevaron al descubrimiento del electrón (1897), la radioactividad de Becquerel y de Pierre y Marie Curie, y varios otros. Los diagramas e ilustraciones de Zegers en este artículo de los Anales eran particularmente interesantes.

A pesar que hacia fines del siglo XIX el interés por la ciencia había crecido y Chile se interesaba cada vez más por lo que acontecía en distintas disciplinas en Europa y EE.UU., las universidades chilenas de esa época eran principalmente instituciones docentes.

\section{UN PRIMER INTENTO DE ORGANIZAR EL DESARROLLO CIENTÍFICO}

El primer intento por transformar las universidades en instituciones que hicieran investigación además de docencia se realizó hacia fines de la década de los 20. Este impulso fue incentivado por Ramón Salas Edwards [5], Gustavo Lira Manso (Rector de la Universidad de Chile y Ministro de Educación) y otros. En esa época se crearon, al menos en el papel, los primeros programas de magíster y de doctorado. Es interesante ver la participación activa de varios chilenos (entre ellos Ramón Salas y Luis Zegers) en la serie de Congresos Panamericanos de Ciencia que se desarrollaron en distintas partes del continente entre 1898 y 1916 (ver, e.g., [4].

Se creó el Instituto de Ciencias de Chile, institución de muy corta duración creada en esa época para albergar el desarrollo de investigaciones científicas en su interior. Por primera vez se estableció un programa para que los jóvenes promisorios egresados de las universidades chilenas fueran en esos años a seguir estudios de postgrado a EE.UU. Se invitó a científicos notables, entre ellos al conocido físico francés Paul Langevin, en 1928. Se contrató a varios profesores europeos de distintas disciplinas científicas. Fue en este período que llegó a Chile (1929) Karl Grandjot, quien obtuvo su doctorado en la Universidad de Göttingen bajo la supervisión de Edmund Landau y luego fue asistente del matemático británico John Littlewood. Karl Grandjot jugó un papel crucial en el desarrollo de las matemáticas y de la ingeniería en Chile, en particular en la Facultad de Ciencias Físicas y Matemáticas, el Pedagógico de la Universidad de Chile, la Escuela de Ingeniería y la Escuela de Arquitectura de la Universidad Católica. Grandjot fue director del DICTUC de la UC y en esa posición, en 1945, construyó el primer computador análogo en Chile.

Lamentablemente, el "crash" económico y político de esos años paralizó este primer esfuerzo por establecer la investigación científica en nuestras universidades. 


\section{CIENCIA, LA FRONTERA SIN LÍMITE}

Sin lugar a dudas la segunda guerra mundial fue un drama horrible, quizás el tiempo de mayor sufrimiento en la historia de la humanidad, en gran parte provocado por la torpeza de los líderes de los países vencedores de la primera guerra (otra gran tragedia). La parte positiva es que este sufrimiento enorme hizo apreciar la paz y la colaboración entre los países. A pesar de toda la tragedia y de los serios pero estúpidos conflictos actuales, el período de posguerra que nos ha tocado vivir ha sido el período más pacífico y de mayor progreso de la historia de la humanidad. La ciencia ciertamente ha jugado un papel crucial en este desarrollo.

Hacia el final de la segunda guerra el entonces Presidente de los EE.UU., Franklin Delano Roosevelt (FDR) le encargó a su asesor científico, Vannevar Bush, que escribiera un informe sobre cómo orientar el enorme esfuerzo científico desarrollado durante la guerra para el beneficio de la sociedad en general en tiempos de paz. Vannevar Bush, ingeniero eléctrico, inventor del computador análogo y precursor de la web, lideró todo el esfuerzo científico de EE.UU. durante la segunda guerra mundial.

Fue precisamente en 1945 que Vannevar Bush escribió el trascendental ensayo "Science, the endless frontier", que luego del fallecimiento de FDR presentó al Presidente Truman. Este ensayo tuvo un efecto inmediato en EE.UU. A raíz de él se crearon la National Science Foundation y la Fundación Fulbright, y las universidades norteamericanas incrementaron fuertemente sus plantas académicas y sus recursos de investigación. Los adelantos científicos de la segunda mitad del siglo XX, probablemente los más acelerados en la historia de la humanidad, cambiaron completamente, en una o dos generaciones, nuestro modo de vida.

El ensayo de Vannevar Bush también tuvo profundas repercusiones en América Latina en general y en Chile en particular. A principios de los 50 visitó Chile, que entonces tenía siete universidades tradicionales (U. de Chile, PUC, UTE, UTFSM, PUCV, U. de Concepción y U. Católica del Norte), una misión de la National Academy of Sciences de EE.UU., misión que hizo varias recomendaciones. Se crearon los primeros institutos de ciencia básica, en física, matemáticas, biología y química; se establecieron las primeras direcciones de investigación en las universidades chilenas; se estableció, en 1957, la Ley de Financiamiento de las Universidades y en la década de los sesenta se crearon la Academia Chilena de Ciencias, cuyo primer presidente fue Gustavo Lira, y CONICYT. En las siete universidades entonces existentes en Chile se hicieron esfuerzos para enviar jóvenes a Europa y EE.UU. a seguir estudios de posgrado. Se contrataron los primeros profesores de jornada completa con la idea de que realizaran al mismo tiempo las tareas de docencia y de investigación.

El rector Juan Gómez Millas, cuyo nombre lleva el campus donde reside la Facultad de Ciencias de la Universidad de Chile, jugó un rol fundamental en 
este período. Hacia fines de los 50 y principios de los 60 se crearon las primeras Licenciaturas en Física en la U. de Chile, la U. de Concepción y la Universidad Católica de Chile, todas las cuales han tenido un desarrollo destacado por ya más de 50 años. Y en 1965 se creó la Facultad de Ciencias de la Universidad de Chile, en un principio inserta en el campus Beauchef de la Universidad de Chile, para luego trasladarse a su lugar definitivo en el campus Juan Gómez Millas. Distinguidos científicos de todas las áreas de la ciencia han egresado de la Facultad de Ciencias, muchos de los cuales han desarrollado carreras académicas brillantes tanto en Chile como en el extranjero.

El efecto de este esfuerzo notable en la historia de Chile, quizás no completamente aquilatado, ha tenido consecuencias importantes en nuestra vida actual. Solo a modo de ejemplo, en todos los gobiernos de los últimos 30 años, independientemente de quiénes hayan conformado la coalición gobernante, ya no se concibe que los ministros de Hacienda y gran parte de sus equipos económicos no tengan un doctorado en economía. Esto puede pasar inadvertido, pero ha traído un crucial ordenamiento en el desarrollo económico de nuestro país. Lo mismo ha ocurrido en el desarrollo de algunas actividades industriales claves de nuestra economía, entre ellas la minería, la industria agrícola y la industria vitivinícola.

Durante los últimos 35 años se ha generado una expansión importante en la actividad científica chilena. En la década de los ochenta se creó FONDECYT, que ha jugado un papel fundamental en el financiamiento de la ciencia en nuestro país. En la misma época se creó la Fundación Andes, hoy desaparecida, la cual jugó, en parte, un papel ejemplar para el financiamiento de la ciencia. En la década de los noventa se crearon los primeros programas asociativos que dieron origen a grupos de investigación de mayor tamaño en distintas áreas de la ciencia; programas como FONDEF, para tratar de establecer vínculos con las empresas; y la Iniciativa Científica Milenio, también focalizada en el desarrollo de programas asociativos. Desde hace poco más de 25 años se ha impulsado un importante y creciente programa de becas de posgrado, que debiera dar un impulso adicional al desarrollo científico chileno.

\section{EL SIGUIENTE PASO}

Actualmente estamos ante una interrogante interesante, cuyo desenlace va a marcar el futuro desarrollo del país. ¿Necesitamos desarrollar ciencia solo como un aporte cultural, como parte de un plan para fortalecer la enseñanza superior, o necesitamos tener un fuerte desarrollo científico como herramienta indispensable para un desarrollo económico y social de nuestro país? Esta disyuntiva ha sido planteada en Chile repetidamente desde fines del siglo XIX, como vimos anteriormente, 
pero nunca se habían dado las condiciones de desarrollo, tanto científico como económico, para plantearlo como una posibilidad real y apremiante de contestar. La situación chilena no es única. Ella ha sido discutida con frecuencia a nivel internacional e incluso hay diversas corrientes de pensamiento relacionadas con esta interrogante (e.g., el Schumpeterismo y muchas otras).

Basándome en la experiencia contemporánea de los países desarrollados no me cabe duda de que el desarrollo científico y su interrelación con el desarrollo industrial es una herramienta indispensable para asegurar un desarrollo económico y social. Si aceptamos este hecho surge una pregunta aún más importante: ¿qué acciones hay que tomar para fortalecer este desarrollo? La respuesta no es fácil, pero ciertamente los puntos más importantes para que este desarrollo se produzca son tres: i) el primero es adoptar políticas de Estado de largo plazo que den estabilidad a este desarrollo; ii) crear condiciones de fuerte liderazgo para poder llevar a cabo este desarrollo; iii) seguir fortaleciendo la educación científica y técnica al más alto nivel posible. Estoy seguro de que si aprovechamos las condiciones actuales de desarrollo, si creamos un ambiente de optimismo como el que indujo Vannevar Bush con su famoso ensayo, "Science, the endless frontier", y si creamos un liderazgo adecuado, podremos alcanzar a mediano plazo un desarrollo económico y social sustentable basado en el desarrollo científico de nuestro país.

\section{REFERENCIAS}

[1] Benguria, Rafael. El rol de la Universidad en el desarrollo cientifico de Chile, Inauguración del Año Académico, viernes 25 de Marzo de 2011, Archivo Histórico, Pontificia Universidad Católica de Valparaíso.

[2] De Vos Eyzaguirre, Bárbara. El surgimiento del paradigma industrializador en Chile, 1875-1900, Ediciones de la DIBAM, Santiago de Chile, 1999.

[3] Etcheverry, María, Porter, Carlos. La "Societé Scientifique du Chili" y las "Actes de la Societé Scientifique du Chili", Revista Chilena de Historia Natural 62, 129-147 (1989).

[4] Fernos, Rodrigo. Science still born, the rise and impact of the Pan American Scientific Congresses, 1898-1916. iUniverse Inc., Lincoln, NE, 2003.

[5] Salas Edwards, Ramón. Escurrimiento variado del agua de los canales, con Prólogo de Cristián Escauriaza y Jorge Gironás, Biblioteca Fundamentos de la Construcción de Chile, volumen 78, Cámara Chilena de la Construcción, PUC, Biblioteca Nacional, Santiago de Chile, 2011.

[6] Serrano, Sol. Universidad y nación, Chile en el siglo XIX, Editorial Universitaria, Santiago de Chile, 1994. 